e-Migrinter

$17 \mid 2018$

Enseigner les migrations internationales

\title{
Regard croisé sur les mineurs non accompagnés étrangers : Récits de vie et pratique d'intervention
} Compte-rendu de la journée d'étude organisée à l'université de Lille-I le 8 mars 2018

Solene Cadart-Mariage et Kevin Sardella

\section{CpenEdition}

\section{Journals}

Édition électronique

URL : https://journals.openedition.org/e-migrinter/1183

DOI : $10.4000 /$ e-migrinter. 1183

ISSN : 1961-9685

Éditeur

UMR 7301 - Migrinter

Référence électronique

Solene Cadart-Mariage et Kevin Sardella, «Regard croisé sur les mineurs non accompagnés étrangers : Récits de vie et pratique d'intervention », e-Migrinter [En ligne], 17 | 2018, mis en ligne le, consulté le 20 mai 2021. URL : http://journals.openedition.org/e-migrinter/1183 ; DOI : https://doi.org/ 10.4000/e-migrinter. 1183

Ce document a été généré automatiquement le 20 mai 2021.

Tous droits réservés 


\section{Regard croisé sur les mineurs non accompagnés étrangers : Récits de vie et pratique d'intervention}

Compte-rendu de la journée d'étude organisée à l'université de Lille-I le 8 mars 2018

Solene Cadart-Mariage et Kevin Sardella

\section{RÉFÉRENCE}

Cadart-Mariage, Solene ; Sardella, Kevin (2018) Compte rendu de la journée d'étude Regard croisé sur les mineurs non accompagnés étrangers : Récits de vie et pratique d'intervention, Lille, Université de Lille-I

1 Le 8 mars 2018 s'est tenue la journée d'étude organisée chaque année par les étudiants de première année de master de sociologie à l'université de Lille. La thématique de cette année, axée sur les phénomènes migratoires, s'est plus spécifiquement concentrée sur la situation des Mineurs Isolés Étrangers (MIE).

2 Ce sujet a eu pour atout d'être placé sous le signe de la pluridisciplinarité et de traiter la problématique à l'aune des différentes spécialités proposées par la formation de master de sociologie (la ville, la santé, le travail et les associations). Cette approche a permis à la promotion de participer au développement de ce projet et de s'en enrichir en retour sur le plan théorique et méthodologique. En outre, le compte-rendu de cette journée partage avec le laboratoire MIGRINTER un objet d'étude analogue articulé autour des questions de migrations. 


\title{
Parcours et expériences des Mineurs Isolés Étrangers accueillis en France
}

\author{
[Intervention de Sarah Przybyl, géographe, post-doctorante à l'UMR MIGRINTER]
}

3 À partir de sa thèse, Sarah Przybyl (2016) propose une analyse des parcours migratoires des mineurs autour de deux séquences pour montrer que leur migration a ses propres logiques. La première s'étend des préparatifs du départ jusqu'à leur arrivée en France. Son exposé montre qu'au préalable, certains jeunes ont eu des expériences de mobilités antérieures à leur émigration vers la France. Ces trajectoires participent ainsi à la constitution de ressources expérientielles relatives au "savoir-migrer ", lesquelles seront alors mobilisées dans les préparatifs comme dans les déplacements au cours de la migration. La préparation du voyage demande alors la constitution et la mobilisation d'un ensemble de ressources (matérielles et immatérielles). La chercheuse distingue trois types de voyage renvoyant à des formes d'expériences spécifiques :

41 - Le premier est une "mobilité instantanée " par voie aérienne, permettant à ces jeunes de parcourir une grande distance et de quitter leur pays légalement en étant inscrit sur le passeport d'un passeur désigné comme tuteur. Par sa rapidité et sa relative sécurité, le trajet en avion est peu évoqué par les jeunes, occupant une place négligeable dans leur discours, contrairement aux représentations dominantes sur la dimension dramatique du voyage et la longueur du trajet vécu. Ici, le rapport à l'espace parcouru le temps du vol se caractérise par une perte des repères spatiaux et temporels (les territoires traversés, la distance). Néanmoins, une fois l'atterrissage effectué, cette mobilité est souvent interrompue par l'abandon du jeune à l'aéroport dans des pays limitrophes à la France (Italie ou Espagne), lequel devra alors ré-impulsé son voyage seul.

52 - Le second est une «migration par étapes » se caractérisant par l'usage successif de divers moyens de transport pour traverser plusieurs frontières terrestres et/ou maritimes. Ce voyage est organisé en amont par la famille s'assurant que le mineur sera escorté par un passeur dans les pays d'escale. Bien qu'il soit planifié, ce périple demeure incertain car il se fait le plus souvent clandestinement et dans une alternance de périodes de mobilité et d'immobilité, (paradoxalement, l'immobilité est constitutive de ce phénomène migratoire : elle est perçue par ces jeunes comme un temps d'attente, de repos et comme un moyen d'acquérir des ressources diverses à une étape donnée du voyage en vue de poursuivre le périple). En termes d'expérience, deux phases se distinguent. La première se fait en compagnie des passeurs : les enquêtés sont moins prolixes parlant rarement en leur nom de cette période. La seconde phase est liée à l'abandon du jeune, impliquant une forme d'autonomisation forcée où il devient alors acteur et seul responsable de sa migration.

63 - Le troisième est un voyage « bricolé » plus chaotique, se faisant aussi par étape, mais s'en distinguant car il comporte des temps plus longs d'arrêt liés aux conditions improvisées et précipitées du départ. Cependant, cette migration n'est pas aléatoire et se construit au fil du trajet en fonction des contraintes et des opportunités. Ici l'immobilité est constitutive de la mobilité, elle est un moyen d'acquérir des ressources matérielles et immatérielles déterminantes pour la suite. Dans ce type de parcours, la minorité et l'isolement sont des variables contraignantes accentuant certains dangers liés aux migrations. En matière d'expérience, même s'il existe un certain brouillage des 
repères lié au traumatisme, le voyage a une place nodale dans les récits car ils en sont les principaux acteurs.

$7 \quad$ La seconde séquence se déroule en France et comprend les différentes prises en charge du mineur par les institutions (juridique, sociale, etc.), lesquelles ne marquent pas la fin du parcours migratoire. L'accès à la protection de l'enfance est en effet ponctué par un ensemble d'étapes et de difficultés à franchir. Après la phase d'évaluation, les jeunes reconnus comme isolés et étrangers sont envoyés vers des centres d'accueil pouvant se situer en milieu urbain ou rural. Par leur localisation, les foyers ruraux présentent des spécificités en termes de contraintes et d'opportunités: bien qu'au niveau administratif, il existe parfois une proximité entre la préfecture et ces structures augmentant ainsi les possibilités de régulation à la majorité, et que de nombreuses initiatives citoyennes se multiplient, la migration de la ville vers la campagne peut être vécue comme une rupture sociale et symbolique s'accompagnant d'un sentiment d'incompréhension pour les mineurs.

\section{Protéger les mineurs, contrôler les migrants}

[Intervention de Nicolas Fischer, chargé de recherche au CESDIP]

8 Au cours de sa thèse, Nicolas Fischer a réalisé une enquête qui a permis de mettre en lumière des tensions à l'œuvre dans l'orientation du parcours des mineurs isolés. Leur trajectoire se trouve marquée par deux postures: celle d'un étranger, susceptible d'inspirer de la méfiance, et celle d'un mineur vulnérable que les pouvoirs publics doivent protéger. Ce double statut influe sur la représentation que les magistrats se font d'eux et impacte donc directement leur avenir. La configuration-même des audiences témoigne de l'importance singulière qui leur est accordée : contrairement à celles des adultes, elles ont lieu dans une salle annexe au tribunal. Cette salle, plus petite et plus intime entend proposer un cadre plus approprié à des mineurs. Par ailleurs, des circonstances atténuantes sont plus facilement attribuées à leurs comportements. Alors que chez les Juges des libertés (JDL) la falsification des papiers sera immédiatement perçue sous l'angle du délit de la part d'un adulte, elle sera envisagée avec bien plus d'empathie et d'acceptation de la part d'un mineur qui se serait sans doute vu contraint de rivaliser d'ingéniosité dans le seul but d'assurer sa protection.

9 La présence obligatoire d'un administrateur ad hoc par individu mineur renforce d'autant plus cette dimension de vulnérabilité. De même, les trajectoires personnelles des intervenants tendent pour beaucoup à souligner cet aspect qu'il s'agisse par exemple d'une enfance souvent difficile pour les administrateurs ad hoc investis dans la Croix-Rouge française, ou bien d'une carrière précédemment universitaire disposant l'une des juges enquêtées à s'opposer systématiquement au principe de répression dans le traitement réservé aux migrants. Quelques profils de magistrats, notamment favorables aux principes d'action des forces de l'ordre, se montrent toutefois réfractaires à cette représentation.

10 Le poids de la trajectoire personnelle des acteurs en présence participe à la constitution d'une arène émotionnelle au cœur de l'audience. À cela contribuent également la proximité physique des intervenants déjà mentionnée ci-dessus, mais aussi, fréquemment, la proximité relationnelle de magistrats qui, se connaissant les uns les 
autres, peuvent prédire leurs réactions réciproques ou témoigner de signes de complicité.

11 Du fait de la prédominance de l'angle de la vulnérabilité sous lequel ils sont perçus, les mineurs sont souvent remis en liberté, confiés à la protection de l'enfance ou aux personnes qui se disent être leurs proches. Malgré tout, des zones d'incertitudes et autres problématiques subsistent autour de leur situation : peut-on systématiquement se fier aux personnes qui, précisément, se disent prêtes à les prendre en charge ? La menace de l'enrôlement au sein de réseaux criminels plane parfois grandement autour de ces mineurs. Est-il pertinent de placer les jeunes de cet âge prétendu sous la tutelle d'un administrateur ad hoc alors qu'ils sont a priori à même de s'exprimer en leur nom? Autant de questions qui reviennent systématiquement face à une multitude de cas et ne font que renforcer cette tension perpétuelle entre deux postures irréconciliables que se voient attribuer les mineurs isolés étrangers.

\title{
Accès à l'autonomie des Jeunes Isolés Étrangers
}

\author{
[Intervention de Lucy Marquet, démographe à l'INED et au CLERSE]
}

12 Bien que non pensée initialement pour traiter de questions relatives à la migration, l'enquête Étude Longitudinale sur l'accès à l'Autonomie des jeunes en Protection de l'Enfance ${ }^{1}$ (ELAP) réalisée par l'INED à partir de 2013 invite à s'arrêter sur les données qu'elle regroupe récoltées en trois vagues (2013, 2014 et 2015) concernant les Jeunes Isolés Étrangers (JIE) - qui incluent les Mineurs Isolés Étrangers (MIE) et les jeunes majeurs de 18 à 21 ans - qui représentent $29 \%$ de leurs enquêtés initiaux.

13 Plus précisément, cette recherche permet de s'intéresser à la façon dont ces jeunes sont pris en charge par l'Aide Sociale à l'Enfance (ASE) dans cinq départements d'Île-deFrance et dans les deux départements de l'ancien Nord-Pas-de-Calais. Sur les sept départements, tous connaissent une surreprésentation des jeunes placés par rapport aux autres départements français.

Le profil socio-démographique des JIE enquêtés apparaît rapidement différent de celui des autres jeunes placés: si ces derniers ont des profils sociologiques multiples (sexe, âge), la majorité des JIE sont placés à partir de 15 ans et sont, pour 88 \% d'entre eux, des garçons. Les familles dont ils sont issus divergent également assez fortement. Si dans la population générale française, $80 \%$ des jeunes de 17 ans ont encore dans leur entourage leurs deux parents, ce n'est le cas que pour $36 \%$ des jeunes placés non-MIE, et pour $25 \%$ des MIE placés. Par ailleurs, les jeunes placés de parents nés en France font pour la plupart partie d'une fratrie assez importante : $43 \%$ d'entre eux ont entre 3 et 5 frères et sœurs, $24 \%$ en ont 6 , tandis que chez les JIE, la proportion d'enfants uniques est bien plus importante. Enfin, bien que les systèmes scolaires soient difficilement comparables à l'international, on relève que $45 \%$ des MIE interrogés ont au moins un parent qui n'a pas été scolarisé. À titre de comparaison, Lucy Marquet rappelle que $38 \%$ des jeunes étrangers arrivés en France accompagnés de leurs parents ont au moins un parent qui a fait des études supérieures.

15 Les efforts de l'ASE vis-à-vis des MIE se concentrent sur leur protection par l'intermédiaire de l'insertion par le logement et la re-scolarisation. D'une part, $53 \%$ d'entre eux déclarent en effet avoir séjourné au moins une nuit de leur vie dans un lieu impropre à la résidence (rue, squat, etc.). Leur hébergement dans un lieu salubre se 
trouve donc au cœur de leur prise en charge. Malgré tout, les JIE subissent plus de transferts que les autres mineurs placés, alors même qu'ils arrivent en moyenne bien plus tard. Ainsi, $43 \%$ d'entre eux ont été hébergés dans deux lieux différents, et $25 \%$ dans trois lieux entre 15 ans et 18 ans. Après leur hébergement initial en foyer collectif, les JIE sont placés plus rapidement que les autres en logement autonome - voire à l'hôtel en région parisienne - pour les préparer à l'indépendance. Cela les confronte à beaucoup de solitude. Ils sont également plus nombreux à être éloignés de leur région d'arrivée puisque les travailleurs sociaux privilégient davantage le maintien dans une région pour des jeunes entretenant encore des liens familiaux. Ils se retrouvent ainsi davantage envoyés dans des zones rurales ce qui renforce leur isolement. D'autre part, $94 \%$ d'entre eux sont re-scolarisés, même si les entretiens révèlent que la plupart arrivent en France avec un certain savoir-faire, et que $74 \%$ sont accueillis en CAP-BEP et $16 \%$ en Bac Pro.

16 À leur majorité, il apparaît qu'ils bénéficient davantage de contrats jeune majeur que les autres jeunes placés. En effet, $96 \%$ des MIE y ont eu accès en 2013 alors que $21 \%$ des non-MIE n'ont pu y avoir accès. La condition de poursuite d'études est pratiquement obligatoire pour pouvoir bénéficier du contrat jeune majeur puisqu'elle concerne $82 \%$ des titulaires. En outre, dans la continuité des conditions de logement précédemment mentionnées, les JIE sont fortement poussés vers une autonomie peu désirée : $67 \%$ des fins de contrats jeune majeur des MIE sont causées par un non-renouvellement de la part de l'ASE, tandis que les non-MIE participent en moyenne bien plus à la décision de prendre leur indépendance. Par ailleurs, après le placement, la location indépendante ne concerne que $48 \%$ des non-MIE contre $64 \%$ des MIE. L'hébergement par la famille ou les amis est choisi quant à lui par $42 \%$ des non-MIE contre à peine $7 \%$ des MIE. Pourtant, 86 \% des MIE déclarent n'avoir personne à contacter en cas d'urgence.

Cette étude révèle que la prise en charge des MIE par l'ASE met l'accent la formation, nécessaire pour la régularisation de leur situation à leur majorité, tandis que les dimensions psychologiques et sociales sont délaissées au risque de renforcer leur isolement sur le territoire français.

\section{Échanges avec le public et conclusions}

Au cours de la discussion, plusieurs thématiques constitutives de la problématique des MIE ont été abordées.

Premièrement, des précisions ont été apportées sur la catégorie MIE et ses variantes. Selon Sarah Przybyl, même s'il existe des définitions légales dans d'autres pays de l'UE, elle n'a pas de valeur juridique propre au sein du droit français : c'est une catégorie d'usage qui a pris une certaine légitimité par son utilisation croissante par les divers acteurs (juridiques, administratifs et sociaux) et l'augmentation progressive de cette population. En réalité, ce qui prévaut aujourd'hui, c'est le droit relatif à la protection de l'enfant qui les considère en situation de danger liée à leur minorité, car ils sont privés temporairement de la protection de leur famille. Lucy Marquet insiste néanmoins sur le fait que l'expression d'usage pour les désigner (circulaires et rapports officiels du sénat) a évolué : d'abord désigné comme des «mineurs étrangers isolés » où l'accent était mis sur leur qualité d'étranger, le terme «mineur isolé étranger », puis celui de « mineur non accompagné étranger » a été utilisé plus systématiquement, se référant 
davantage à la privation temporaire de la protection d'un tuteur qu'au statut d'étranger.

Deuxièmement, la discussion sur la place des professionnels intervenant dans la prise en charge de ces jeunes a montré qu'ils entretiennent parfois des rapports ambigus avec les procédures imposées par le département. D'une part, certaines mesures pourront être contournées par les actions de terrain pour des motifs divers (militantisme, inefficacité, etc.). D'autre part, les pratiques des travailleurs sociaux s'articulent avec les logiques des politiques de contrôle de plus en plus contraignantes, par exemple des structures mandatées par le département réalisent des évaluations en vue de se prononcer sur la minorité du jeune.

Troisièmement, cet objet d'étude implique un rapport singulier du chercheur à son terrain conduisant à des régimes d'engagement particulier. Même dans les approches quantitative, Lucy Marquet indique que la production de statistiques, notamment dans des domaines où il existe peu de données, interrogent le chercheur sur la façon dont elles vont être utilisées, ainsi que les effets qu'elles génèreront sur les dispositifs existants et sur la population étudiée. La prise de position peut aussi apparaître face à la complexité du terrain. Par la déconstruction des discours institutionnels, Nicolas Fisher a d'ailleurs noté une tension traversant les dispositifs d'intervention (dont ceux de renvoi) entre l'État de droit, la prévention et la répression. Cette tension s'accompagne d'une prise de conscience de leur inefficacité et des effets pervers qu'ils génèrent (précarisation, criminalisation des frontières).

\section{BIBLIOGRAPHIE}

Fischer, Nicolas (2012) Protéger les mineurs, contrôler les migrants. Enjeux émotionnels et moraux des comparutions de mineurs enfermés aux frontières devant le Juge des libertés et de la détention, Revue française de sociologie, vol. 53, n 4, pp. 689-717.

Przybyl, Sarah (2016) Au cœur du dispositif de protection : processus d'adaptation et de renégociation du projet migratoire des mineurs isolés étrangers à Paris, Poitiers, Université de Poitiers, 501 p., Th. Doct : Géogr. : Poitiers : 2016.

\section{NOTES}

1. Réalisée en deux vagues, cette enquête s'est donnée pour objectif de suivre le parcours de jeunes accueillis à l'ASE (conditions de vie et de sortie du dispositif) afin d'espérer orienter les futures politiques publiques dont ils pourront faire l'objet. 
INDEX

Index géographique : France

Mots-clés : mineurs isolés, catégorisation, intégration sociale, attitude à l'égard des étrangers

\section{AUTEURS}

\section{SOLENE CADART-MARIAGE}

Master 1 de sociologie, Université de Lille 1 solene.cadart-mariage@outlook.fr

\section{KEVIN SARDELLA}

Master 1 de sociologie, Université de Lille 1 sardella.kevin1@gmail.com 